\title{
A shared voice: engaging First Nations and Inuit communities in the development of culturally appropriate asthma and allergy education materials and resources for youth and their families
}

\author{
Oxana Latycheva, Christine Hampson, Mark Greenwald*, Sabrina Panetta, Rupinder Chera \\ From Canadian Society of Allergy and Clinical Immunology Annual Scientific Meeting 2010 \\ Victoria, Canada. 3-6 November 2010
}

\section{Background}

The prevalence of asthma and associated allergies is higher in First Nations and Inuit communities than in the general Canadian population. The main project purpose was to assess the relevance of existing asthma education materials and resources to First Nations and Inuit communities, and identify how these materials could be adapted to be culturally appropriate.

\section{Materials and methods}

Three sources of data were used to compile the findings. First, 68 asthma assessment packages and questionnaires were completed by Aboriginal community members. Second, five webinars with $\mathbf{5 6}$ participants in total were conducted. Third, an Advisory Group was created to discuss potential barriers to receiving asthma education.

\section{Results}

Aboriginal community members valued interactivity, visual features of materials, and personal interaction. Newly developed materials should focus on practical and lifestyle issues of asthma management. Participants also preferred a combination of traditional printed and digital resources. Cultural relevance could be improved by including images related to Aboriginal culture, featuring personal stories, and making materials available in Aboriginal languages.

\section{Conclusions}

The findings support five key recommendations. Firstly, there should be a focus on the development of culturally appropriate asthma educational materials and resources. Secondly, implementation of asthma educational activities for children should be a priority. Thirdly, education should target broader community members to increase community awareness of asthma. A fourth recommendation is to ensure appropriate access to educational resources in the communities. Finally, it is crucial to continue engaging First Nations and Inuit community members in the development/adaption of asthma educational materials and community-based programs.

\section{Acknowledgements}

We would like to extend our thanks to the First Nations and Inuit Health Branch (FNIHB), Health Canada for providing funds for this important project.

Published: 4 November 2010

\section{doi:10.1186/1710-1492-6-S2-P32}

Cite this article as: Latycheva et al:: A shared voice: engaging First Nations and Inuit communities in the development of culturally appropriate asthma and allergy education materials and resources for youth and their families. Allergy, Asthma \& Clinical Immunology 2010 6(Suppl 2):P32.

\footnotetext{
* Correspondence: drmarkgreenwald@rogers.com

The Asthma Society of Canada, Toronto, Ontario, Canada, M2N 6K1
} 